\title{
Pelvic Inflammatory Disease, A Perturbing Trigger to Infertility: A Systematic Review
}

\author{
Dr. Gul E Nasreen Syyeda Khadija Dr. Saadia Fatima \\ University Institute of Radiological Sciences and Medical Imaging Technology (UIRSMIT)University of \\ Lahore, Pakistan
}

\begin{abstract}
Introduction: Pelvic inflammatory disease is the term used comprehensively for cervicitis, endometritis, salpingitis, pelvic peritonitis, and tubo-ovarian abscess. Diagnosis is initially by clinical assessment, confirmed by laboratory investigations and imaging modalities. PID is usually ignored by patients and clinicians eventually leading to infertility. Purpose: The goal of this systematic review is to evaluate co relation of PID with infertility and role of imaging modalities for diagnosis. Method: During October 2020 we searched Google scholar, pub med, Medscape research articles from 2010 -2020. Conclusion: Pelvic inflammatory disease is second most common cause of infertility while diagnostic ultrasonography plays a limited role to diagnose.
\end{abstract}

Keywords: Pelvic inflammatory disease, infertility, endometritis, hydro salpinx, Tubal factor infertility and Sonographic evaluation.

DOI: $10.7176 / \mathrm{JHMN} / 82-08$

Publication date: November $30^{\text {th }} 2020$

\section{Introduction:}

Pelvic Inflammatory Disease is collectively termed for infections involving female genital tract comprising of cervix, uterine endometrium, ovaries, fallopian tubes, and pelvic peritoneum. Organisms thought to be culprit are Chlamydia trachomatis and Neisseria gonorrhea, which are cause of sexually transmitted diseases (STI,) but clinical disease is not associated with only these organisms. It is difficult to diagnose Pelvic Inflammatory Disease due to variable clinical presentations ranging from subclinical PID to severe disease that may need surgical interventions. Presentation of acutePID without clinical signs and symptoms is defined as subclinical PID. CDC 2015 Sexually Transmitted Diseases Treatment Guidelines gave minimal clinical criteria to offer conjectural treatment for PID to sexually active woman or woman at risk for STIs who had been through lower abdominal or pelvic pain and on clinical examination either cervical motion tenderness or uterine tenderness or adnexal tenderness was experienced. Additional standards include oral temperature more than 101F (38.3C), friable cervix, cervical mucopurulent discharge. Vaginal fluid showing abundance of white blood cells on microscopy. Erythrocyte sedimentation rate and C-reactive proteins with raised values, Neisseria gonorrhea and Chlamydia trachomatis documented by labortary.last but not the least is Specific diagnostic criteria comprising of Transvaginal ultrasound or magnetic resonance imaging exhibiting thickening of fluid filled tubes with or without free fluid in pelvis, or tubo ovarian complex, pelvic infections manifested by Doppler,Laporoscopic findings revealing PID and evidence of endometritis on histopathology of endometrial biopsy.(1)

Mostly bacteria are the cause of inflammation in PID [3]. Chlamydia trachomatis, Neisseria gonorrhea, Streptococcus species, Enterococcus faecalis are the responsible microorganisms.

PID evolved subsequently in About 10\% of women previously infected by Chlamydia trachomatis. Bacterial vaginosis (BV) may also be responsible for tubal 1 factor infertility by its mechanism of reduction of lactobacilli, raising vaginal $\mathrm{pH}$ and stimulating growth of anaerobic vaginal organisms ${ }^{(2)}$.

Females considered at high risk of PID are of young age (30 years), single status, multiple sex partners, lower socioeconomic status, with reluctance of seeking medical advice, use of intrauterine contraceptive device, previously experienced interventionary methods of endometrial biopsy or hysteroscopy or HSG (HysteroSalpingography).

PID is a noteworthy public health problem. most of the cases go unreported and about two third of cases remained un noticed. We are unable to determine the actual incidence of PID due to lack of reliable diagnostic techniques as well as asymptomatic nature of the disease. In United States more than one million women are affected by an episode of acute PID every year [4]. Approximately, more than 100,000 women have to put up with infertility each year ${ }^{(3)}$.

\section{Methods:}

Search strategy

We searched Google Scholar, PubMed, Medline, Medscape, Cochrane. All words like Pelvic Inflammatory Disease, Infertility, sonographic evaluation, hydro salpinx, endometritis, tubal factor infertility were used. 


\section{Study selection}

We studied 30 articles and then excluded prospective, case control and case reports from data. We applied additional methodological filter for paper selection and searched articles published between $2010-2020.19$ articles were selected for systematic review. Articles containing data from South Asia were preferred.

\section{Systematic Review}

In 2010 a study was conducted by Nousheen Aziz for Laparoscopic Evaluation of female factors in Infertility by a case series of all primary and secondary infertile women. It revealed $21.9 \%$ of primary and $33.3 \%$ of secondary infertility is due to Tubal Blockage (PID) (4)

In 2013 Shraddha K Shetty investigated 50 women with primary and secondary infertility for tubal disease by laparoscopy and concluded $44 \%$ of primary and $50 \%$ of secondary infertility is due to nonspecific pelvic inflammatory disease. The study revealed that ensuing incidents of PID subsequently increased the risk of infertility ${ }^{(5)}$. in 2014 zhao w.-h. · hao $\mathrm{m}$. conducted a retrospective analysis to spot the reason of pelvic inflammatory disease (pid) and infertility in north china. shanxi,

He stipulated 4,540 cases of infertility 1922 (42.3\%) cases were distinguished with PID. His results showed incidence for nonspecific PID was $57.9 \%$ and $42.1 \%$ for Genital Tuberculosis. he concluded tubal infertility is eventual outcome of PID ${ }^{(6)}$.

In 2015 a retrospective study was mediated by Babita Kumari in the Department of Microbiology, Patna Medical Collage, Patna. She selected 150 cases for this study from wards and OPD of the Department of Obstetrics and Gynecology PMCH, Patna in time period of 13 months i.e. August 2013 to September 2014.Cases with symptoms of raised temperature, chronic pelvic pain, and abnormal vaginal discharge were selected. women seeking treatment for infertility were also included who were of age group 26-35 yrs. Aspirate from pouch of Douglas and High vaginal swab were taken to examine. Study revealed that $48.24 \%$ cases were reported for PID and $55.55 \%$ for infertility illustrating strong relation with the optimum reproductive life ${ }^{(7)}$.

In 2015 Jasmina Begum conducted a retrospective study of 135 patients aged of $19-40$ years at Department of Obstetrics and gynecology MGMCRI Puducherry by combined Hysterolaproscopy and 2nd commonly abnormality detected was Pelvic inflammatory disease i.e. $18.5 \%{ }^{(8)}$

In 2016, Chayachinda Chenchit conducted a retrospective cohort study in patients hospitalized with pelvic inflammatory disease (PID) and predicted the prevalence of adverse reproductive outcomes in them. The criteria for this study was sexually active aged 14-40 years' women admitted with PID or Tubo ovarian abcess for a period of 7 years from January 2004 to December 2011.

69 [IQR 51-93] months duration follows up unfolded that out of 94 patients, $24(25.5 \%)$ linked up to the criteria of infertility, $15(16.0 \%)$ suffered recurrent PID and $13(13.8 \%)$ experienced chronic pelvic pain. For live births the only negative predictor factor was TOA (aOR $0.23,95 \% \mathrm{CI} 0.07-0.79, p=.019){ }^{(9)}$.

A retrospective study was conducted by Sairem Mangolnganbi Chanu, for duration of 2 years i.e. July 2014 to June 2016.He evaluated 151 patients and presumed primary and secondary infertility $58.28 \%$ and $41.72 \%$ respectively. Abnormal findings were assessed in $37.5 \%$ of primary infertility group and $49.2 \%$ of secondary infertility group. Adnexal adhesions (pelvic inflammatory disease) were most common finding (10).

In 2017 KHOURI described Ultrasound is considered first line imaging modality in the evaluation of fallopian tubes and techniques to distinguish between chronic and acute pelvic inflammatory disease as well as infertility. He also mentioned employing modern ultrasonography such as color Doppler and three dimensional imaging and salpingocentesis in clinical practice ${ }^{(11)}$.

Dr Rahat Akhtar conducted a cross sectional study in department of Obstetrics and Gynecology Nishter Hospital Multan in 2017 on sub-fertile women to know THE factors frequently leading to tubal impetency. Laparoscopy was assigned for evaluation and $27.4 \%$ patients of infertility were summed up due to Pelvic inflammatory disease ${ }^{(12)}$

In 2017, Nafeesa Binti Hussain from BGC Trust Medical College Department of Obstetrics \& Gynecology, Chittagong in 2017 conducted a study on infertile women of reproductive age over one-year period. It was an interesting effort to diarize TVS findings in patients complaining infertility. Among the 100 cases $75 \%$ cases were documented with primary infertility and $25 \%$ with secondary infertility. Out of 100 cases undergone TVS study illustrated results were as following, chronic pelvic inflammatory disease was $14(14 \%)$ of cases, free fluid in pelvic/abdominal cavity was in $7(7 \%)$ cases, while $5(5 \%)$ showed endometritis, tubo-ovarian mass was $2(2 \%)$ and $2 \%$ share was from intrauterine and intra pelvic adhesions ${ }^{(13) .}$

C Casper D J den Heijer in 2019 conducted retrospective study at Clinical Practice Research Datalink GOLD database, 2000-2013.12- 25 years aged women were specified who had previous Chlamydia trachomatis (CT) diagnosis. Objective of the study was to estimate the risk of pelvic inflammatory disease (PID) infertility and ectopic pregnancy due to this infection. 857324 women were analogized on the basis Chlamydia Trachomatis test. The study divulged that risk of PID is increased in CT-positive women (adjusted hazard ratio [aHR], 2.36; 95\%

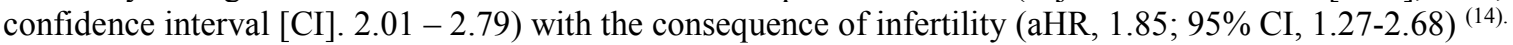


In 2020 Afshan Majeed conducted a study in Quetta that the difficulty to conceive is seen as a major problem among families especially in Pakistan. The study was designed to get the measure of different causes of female infertility in different ethnic groups of Baluchistan, using ultrasonography technique and supporting information. 100 infertile females of the age ranging from 20- 45 yrs were divided into 5 groups with difference of of $5 \mathrm{yrs}$ i.e. $20-25$ and so on. Her study deduced that diagnostic Ultrasonography is worthwhile and instructive to find the causes, analysis and to administer the treatment of female infertility. Study showed that the Tubal factor infertility which is blocking of fallopian tubes, is one the most common cause of females to not conceive and recognized in approximately $30 \%-35 \%$ of women putting up with infertility. The most prevailing rationale of tubal factor infertility is pelvic inflammatory disease (PID) ${ }^{(15)}$.

In 2017 Patrik M Bavoil presented an interesting hypothesis based upon recent, historical and circumstantial evidences that prevalence of Chlamydia trachomatis is increasing due to rising practice of oral sex as the infectious organism produced in GI tract and reached to rectum. It can infect female urogenital tract by its chronic contamination which may lead to serious sequelae of Chlamydia infection in women i.e. pelvic inflammatory disease eventually resulting in tubal factor infertility ${ }^{(19)}$.

\section{Discussion}

PID is a dynamic problem and continuous increase in the number is observed from 2009-2012.in acute cases of PID early diagnosis and intervention is drastically important (17).) Because endometrial secretary transformation is violated in patients with infertility associated with pelvic inflammatory disease ${ }^{(16) .}$

The long term complications of PID were linked with disease severity, delay in treatment and recurrence of infection. Repeated episodes worsens the sequel of PID so important goal is prevention of recurrent PID (17).

Clinicians should get alert for adequate long term care and cure of PID and TOA due to elevated prevalence of its adverse squeal. ${ }^{(8)}$

Chlamydia and gonorrhea are the most common known causes of PID. Annual screening of chlamydia and gonorrhea should be practiced to avert PID that may eventually reduce the burden of infertility ${ }^{(18)}$.

Patient education about personal hygiene, proper sanitation and cleanliness is necessary. Public health interventions to use of adequate measures for intra uterine contraceptive devices and sex education play a role to reduce rate of PID $^{(1)}$.

\section{Conclusion}

Infertility was reported 4 fold higher prevalent among women of 18 -29 years of age group with history of PID ${ }^{(18)}$ so special attention should be paid by health authorities towards its early diagnosis and prevention ${ }^{(3)}$. Such potential cost-effective approaches should be implemented which could be employed in real-world settings ${ }^{(1)}$.

\section{Acknowledgements}

The authors are grateful to the institute of Radiological Sciences and Medical Imaging Technology, University of Lahore.

\section{Compliance with Ethical Standards}

There is no violation of human or animal rights.

\section{Recommendation:}

A strong requisite is documented for further involvement and progress of diagnostic and interventional ultrasonography for the early diagnosis and cure for PID.

\section{Conflict of Interest}

Authors confirm thsat this manuscript has no conflict of interest.

\section{References}

1. Breanne B Das. Pelvic inflammatory disease: improving awareness, prevention, and treatment, 9 ed. Infect Drug Resist, 2016.

2. Dr Madhuri Patil. Non-tuberculous Pelvic Inflammatory Disease and Infertility. AIM (advanced infertility management)

3. Rachana Pachori. the incidence of pelvic inflammatory diseases and associated clinical consequences in reproductive women. world journal of pharmacy and pharmaceutical sciences, 20ssss 16

4. Nousheen Aziz. laparoscopic evaluation of female factors in infertility. journal of the college of physicians and surgeons Pakistan, 2010.

5. Shraddha K. Shetty. laparoscopic evaluation of tubal factor in cases of infertility. international journal of reproduction, contraception, obstetrics and gynecology, 2013. 
6. Zhao W.-H. pelvic inflammatory disease: a retrospective clinical analysis of 1,922 cases in north china, 77 ed. gynecol obstet invest, 2014.

7. Babita Kumari . study of pathogens in high vaginal swab and cul-de-sac aspirate in women with pelvic inflammatory disease and infertility. Kumari International Journal of Contemporary Medical Research 2016; $3(4)$.

8. Jasmina Begum. Combined hysterolaparoscopy as an early option for initial evaluation of female infertility: a retrospective study of 135 patients. International Journal of Reproduction, Contraception, Obstetrics and Gynecology 2015; 4(3).

9. Chayachinda, Chenchit. Reproductive outcomes of patients being hospitalized with pelvic inflammatory disease. - Journal of Obstetrics and Gynecology 2017; 37(2).

10. Sairem Mangolnganbi Chanu. 23Diagnostic Hysterolaparoscopy for Evaluation of Infertility: Our Experience in a Tertiary Care Hospital, J Hum Reprod Sci. 2018; 11(1).

11. OR Khouri. Tubal Disease and Impersonators/Masqueraders. Clinical Obstetrics and Gynecology 2017; 60(1).

12. Dr. Rahat Akhtar1. sub fertile women; frequency of factors leading to tubal blockage evaluated by laparoscopy. The Professional Medical Journal 2017; 24(4).

13. Nafeesa Binti Hussain. Transvaginal Ultrasound Findings Among the Women Presenting with Infertility, Chattagram Maa-O-Shishu Hospital Medical College journal 2017; 16(2).

14. Casper D J den Heijer. Chlamydia trachomatis and the Risk of Pelvic Inflammatory Disease, Ectopic Pregnancy, and Female Infertility A Retrospective Cohort Study Among Primary Care Patients, Clinical Infectious Diseases 2019; 69(9).

15. Afshan Majeed. Diagnosis of oligomenorrhea and amenorrhea in females (infertility) of different ethnic groups of Quetta Baluchistan. Pure and Applied Biology (PAB) 2020; 10(2).

16. Tolibova G.K. Comparative evaluation of morphological criteria of endometrial dysfunction in patients with infertility associated with pelvic inflammatory disease, external genital endometriosis and uterine myoma. Journal of Obstetrician and women Disease JO 2016; 65(6).

17. Pavel Sitnik. Long-term sequelae of pelvic inflammatory diseases. Journal of Education, Health and Sport. 2016; 6(10).

18. Anyalechi. Self-Reported Infertility and Associated Pelvic Inflammatory Disease Among Women of Reproductive Age-National Health and Nutrition Examination Survey, United States, 2013-2016. Sexually Transmitted Diseases 2019; 46(7).

19. Patrik M Bavoil. Does Active oral sex Contribute to Female Infertility? Journal of Infectious Diseases 2017; 216(8). 